\title{
A New Inflammatory Parameter Can Predict Delayed Intracranial Hemorrhage Following Ventriculoperitoneal Shunt
}

\section{Shiwei Li ( $\sim 516760848 @ q q . c o m$ )}

Ningbo Medical Treatment Centre Li Huili Hospital https://orcid.org/0000-0002-2669-5002

Hongcai Wang

Ningbo Chinese Medical Hospital

Feng Li

Medical Imaging Department of Ningbo Medical Center Lihuili Hospital

Maosong Chen

Neurosurgery Department of Ningbo Medical Center Lihuili Hospital

\section{Pandi Chen}

Neurosurgery Department of Ningbo Medical Center Lihuili Hospital

\section{Research}

Keywords: ventriculoperitoneal shunt, delayed intracerebral hemorrhage, neutrophil-to-lymphocyte ratio, hydrocephalus, inflammatory response

Posted Date: November 13th, 2020

DOl: https://doi.org/10.21203/rs.3.rs-104468/v1

License: (1) This work is licensed under a Creative Commons Attribution 4.0 International License. Read Full License 


\section{Abstract}

Background: Delayed intracerebral hemorrhage (DICH) secondary to ventriculoperitoneal (VP) shunt is considered to be a potentially severe event, however, little is known about the risk factors and underlying mechanisms. This study aimed to investigate the association between NLRR (a ratio of postoperative neutrophil-to-lymphocyte ratio to preoperative neutrophil-to-lymphocyte ratio) and DICH secondary to VP shunt.

Methods: We performed a retrospective review of patients who underwent VP shunt between January 2016 and June 2020. Multivariable logistic regression analysis was used to assess the association of DICH and NLRR. Then patients were divided into two groups according to the optimal cut-off point of NLRR, propensity score matching (PSM) method was performed to reconfirm the result.

Results: A total of 130 patients were enrolled and DICH occurred in 29 patients. Elevated NLRR (odds ratio [OR], 2.774; 95\% confidence interval [CI], 1.372-5.609; $\mathrm{P}<0.001)$ and history of craniotomy (OR, 3.505; $95 \% \mathrm{Cl}, 1.040-11.813 ; \mathrm{p}=0.043$ ) were independent risk factors for $\mathrm{DICH}$ secondary to VP shunt. Receiver operating characteristic curve analysis of NLRR showed that area under the curve was 0.832 . The optimal cut off point of NLRR was 2.05 , and the sensitivity was $89.7 \%$, the specificity was $63.4 \%$. Patients with NLRR>2.05 had much higher incidence of $\mathrm{DICH}(\mathrm{OR}, 11.25 ; 95 \% \mathrm{Cl}, 1.35-93.50 ; \mathrm{p}=0.025$; PSM cohort, $\mathrm{n}=82)$.

Conclusions: Our finding suggested that DICH following VP shunt was not a rare complication and elevated NLRR could independently predict DICH. Inflammatory responses might play an important role in the development of DICH following VP shunt.

\section{Background}

Ventriculoperitoneal (VP) shunt is the most common treatment for hydrocephalus ${ }^{1}$, and the placement of VP shunt is one of the routine neurosurgical procedures worldwide ${ }^{2}$. The common complications secondary to VP shunt are shunt obstruction, infection, seizure, subdural hemorrhage and shunt malfunction ${ }^{3,4}$. Mild hemorrhage is frequently observed in the ventricle or in the parenchyma soon after operation ${ }^{5}$, and the rate could be up to $43.1 \%^{6}$. However, the delayed intracerebral hemorrhage (DICH) is considered to be a rare but potentially severe event ${ }^{7}$, the mortality is as high as $50 \%^{4}$. The risk factors and underlying mechanisms of DICH are still not fully elucidated.

A recent study pointed out that the systemic inflammatory responses might be involved in the pathologic process of active cerebral hemorrhage ${ }^{8}$. We hereby assume that inflammatory response is one of the mechanisms associated with DICH following VP shunt. The neutrophil-to-lymphocyte ratio(NLR), as a rapid and economic biomarker of systemic inflammation ${ }^{9}$, has been a dependable predictor of clinical outcome in patients with spontaneous intracerebral hemorrhage ${ }^{10}$ and traumatic brain injury ${ }^{11}$. Considering that the value of NLR is greatly influenced by the basic systemic inflammatory statuses such 
as pneumonia or urinary infection, we proposed a new parameter named NLRR, what is a ratio of postoperative NLR to preoperative NLR. In this study, we sought to test the hypothesis that elevated NLRR is associated with the DICH secondary to VP shunt.

\section{Methods}

\section{Patient Selection}

We performed a retrospective review of patients who underwent VP shunt between January 2016 and June 2020 from the Neurosurgery Department of Ningbo Medical Center Lihuili Hospital. Inclusion criteria were as follows: (1) age $\geq 18$ years; (2) the diagnosis of hydrocephalus was confirmed by clinical symptoms and imaging examination, and VP shunt was performed in our hospital; (3) laboratory tests (Blood routine and coagulation function) within 5 days before VP shunt, and blood routine on the first morning after VP shunt; (4) postoperative brain computed tomography (CT) scan was performed on the first day after operation (later than the postoperative test of blood routine), and at least one CT scan was performed within 5-10 days after operation. The exclusion criteria were as follows: (1) patients on a regimen of anticoagulant or antiplatelet therapy; (2) patients with Ommaya reservoir implantation, the Ommaya tube was directly connected to the shunt pump without ventricular puncture, or the Ommaya tube was removed during the surgery; (3) cranioplasty and VP shunt were performed simultaneously; (4) a revision of the VP shunt; (5) early intracerebral hemorrhage after VP shunt, which was defined as bleeding on the first day after operation.

$\mathrm{DICH}$ was defined as subsequent hemorrhage in the ventricle or the parenchyma along the catheter path which was not found in the CT scan on the first day after operation. The patients enrolled in the study were divided into two groups according to whether or not $\mathrm{DICH}$. The patient flowchart was summarized in Fig. 1.

\section{Data collection}

Demographic characteristics and clinical variables were collected such as: sex and age; history of hypertension and diabetes; history of craniotomy and skull defect; preoperative pneumonia and Glasgow Coma Scale (GCS); primary intracranial lesions including normal hydrocephalus, trauma, spontaneous intracerebral hemorrhage $(\mathrm{ICH})$, tumor and inflammation; hydrocephalus types including low pressure hydrocephalus ( $\mathrm{LPH}$, cerebrospinal fluid pressure $<80 \mathrm{~mm} \mathrm{H}_{2} \mathrm{O}$ ), normal pressure hydrocephalus ( $\mathrm{NPH}$, $80 \mathrm{~mm} \mathrm{H}_{2} \mathrm{O} \leq$ cerebrospinal fluid pressure $\leq 180 \mathrm{~mm} \mathrm{H}_{2} \mathrm{O}$ ), high pressure hydrocephalus (HPH, cerebrospinal fluid pressure $>180 \mathrm{~mm} \mathrm{H}_{2} \mathrm{O}$ ). For the patients with $\mathrm{DICH}$ secondary to VP Shunt, the onset day of hemorrhage, types of hemorrhage, with or without symptom after hemorrhage and the Glasgow outcome scale (GOS) were also collected. Laboratory variables were retrieved from our hospital's database. International normalized ration (INR), prothrombin time (PT), activated partial thromboplastin time (APTT), serum thrombocytes, neutrophils and lymphocytes were collected within 5 days before operation. Postoperative serum neutrophils and lymphocytes were collected on the first morning after 
operation. All of the preoperative and postoperative brain CT scans were obtained, and respectively reviewed by one neurosurgeon and one radiologist who blinded to the detail information (demographic and clinical variables, laboratory data) of patients. The different opinions between them were resolved by consultation. The volume of hematoma was calculated by 3D slicer (version 4.10.2). Besides, postoperative cerebral edema around catheter on the first postoperative CT scan was also collected.

\section{Procedural technique}

All of the patients were implanted with Medtronic Strata Adjustable Pressure Valve Systems, and the initial pressures were collected. The standard technique for VP shunt was employed, and the catheter was placed into the left or right anterior frontal horn via a bur hole at Kocher's point. The postoperative manipulation of valve system was recorded before the occurrence of $\mathrm{DICH}$, or within 15 days after operation of patient without DICH.

\section{Statistical analysis}

SPSS version 26.0 (IBM Corporation, Armonk, New York, USA) and R Software (version 4.0.2) were used for data analysis with statistical significance was defined as $P<0.05$. The Kolmogorov-Smirnov test was used to determine the distributions of continuous variables. The continuous variables with non-normally distributions were analyzed by Mann-Whitney test, presented as the median(50th) with interquartile range (IQR). Categorical variables were presented as number(proportion). Mann-Whitney test was used for ordered categorical variables, and unordered categorical variables were analyzed by Pearsons chi-square test, Continuous correction chi-square test or Fishers exact test. The variables with $\mathrm{P}<0.1$ and variables proposed by previously published articles, were included into multivariable logistic regression analysis to assess the association of DICH and NLRR. The predictive value of NLRR for DICH following VP shunt in patients was evaluated by receiver operating characteristic (ROC) curve analysis. Then patients were divided into two groups according to the optimal cut-off point of NLRR (NLRR $\leq$ cut-off point group and NLRR > cut-off point group). Propensity score matching (PSM) method was performed to adjust for imbalances of patients' characteristics between two groups. Covariates such as sex, age, history of hypertension and diabetes, history of craniotomy and skull defect, preoperative pneumonia and GCS grade, primary intracranial lesion, hydrocephalus type, preoperative PT and APTT, preoperative INR and PLT, puncture site, initial pressure of vale system, brain edema around catheter and postoperative manipulation of valve system were matched at a ratio of 1:1 using a caliper width of 0.2 . The estimation algorithm of propensity score was logistic regression and matching algorithm was nearest neighbor matching. After PSM, 82 patients (NLRR $\leq 2.05$ group: $n=41$, NLRR $>2.05$ group: $n=41$ ) were selected to analysis.

\section{Results}

\section{Characteristics of the patients}


A total of 130 patients underwent VP shunt were included in this study and divided into two groups: nonDICH group $(n=101)$ and DICH group $(n=29)$. Because there were 36 patients with early intracerebral hemorrhage after VP shunt who were excluded, the overall incidence of DICH secondary to VP shunt was $17.5 \%$ (29/166). Characteristics and clinical data were compared between the two groups and shown in Table 1. Most of the data were comparable, except history of craniotomy and preoperative NLR, postoperative NLR, NLRR. 15 patients in the DICH group (51.9\%) presented a history of craniotomy, while the number in the non-DICH group is $34(33.7 \%)(P=0.008)$. Lower preoperative NLR $(2.27$ vs $2.89, \mathrm{P}=$ $0.037)$ and higher postoperative NLR ( 6.08 vs $4.75, P=0.001)$ were observed in patients with DICH secondary to VP shunt. NLRR in the DICH group was 3.44 (IQR 2.33-4.10), which was much higher than the NLRR $(1.69$, IQR 1.15-2.44) in the non-DICH group $(P<0.001)$. 
Table 1

Characteristics and clinical data of the patients

Variables

Demographics

Male sex, $\mathrm{n}(\%)^{\wedge}$

Age (y), median[IQR]

Clinical history, $\mathrm{n}(\%)$

Hypertension $\mathbf{A}$

Diabetes mellitus $\boldsymbol{\nabla}$

Craniotomy ${ }^{\mathbf{A}}$

Skull defect $\boldsymbol{\Lambda}$

Preoperative pneumonia^

Preoperative GCS, median[IQR]

Primary intracranial lesion, $n(\%)^{\square}$

normal hydrocephalus

trauma

$\mathrm{ICH}$

tumor

Inflammation

Hydrocephalus type, $n(\%)$

LPH

NPH
Non-DICH $(n=101)$

$\operatorname{DICH}(n=29)$

P
59(58.4)

$60.0[54.0-67.5]$

34(33.7)

13(12.9)

52(51.5)

24(24.8)

33(32.7)

12[9-15]

7(6.9)

39(38.6)

39(38.6)

14(13.9)

2(2.0)

9(8.9)

75(74.3)
0.236

1(3.4)

17(58.6)

9(31.0)

1(3.4)

1(3.4)

2(6.9)

0.491

0.667

$0.077 *$

0.965

$0.008^{* *}$

0.497

0.598

0.674

0.243

26(89.7)

Abbreviations: GCS, Glasgow Coma Scale; ICH, spontaneous intracerebral hemorrhage; LPH, low pressure hydrocephalus; NPH, normal pressure hydrocephalus; $\mathrm{HPH}$, high pressure hydrocephalus; Pre-PT, preoperative prothrombin time; Pre-APTT, preoperative activated partial thromboplastin time; Pre-INR, preoperative international normalized ration; Pre-PLT, preoperative serum thrombocyte; PreNLR, preoperative neutrophil-to-lymphocyte ratio; Post-NLR, postoperative neutrophil-to-lymphocyte ratio; NLRR, a ratio of post-NLR to pre-NLR.

Mann-Whitney U test; $\boldsymbol{\Delta}$ Pearsons chi-square test; ${ }^{\nabla}$ Continuous correction chi-square test; ${ }^{\square}$ Fishers exact test

${ }^{*} \mathrm{P}<0.1,{ }^{\star *} \mathrm{P}<0.05$ 


\begin{tabular}{|c|c|c|c|}
\hline Variables & Non-DICH $(n=101)$ & $\mathrm{DICH}(n=29)$ & $\mathbf{P}$ \\
\hline $\mathrm{HPH}$ & $17(16.8)$ & $1(3.4)$ & \\
\hline \multicolumn{4}{|l|}{ Laboratory test, median[IQR] } \\
\hline Pre-PT(seconds) & $11.9[11.4-12.6]$ & $11.8[11.2-12.5]$ & 0.503 \\
\hline Pre-APTT(seconds) & $29.7[27.8-32.1]$ & $31.2[29.5-32.5]$ & $0.073^{*}$ \\
\hline Pre-INR & $1.04[1.00-1.10]$ & $1.04[0.96-1.09]$ & 0.544 \\
\hline $\operatorname{Pre}-\operatorname{PLT}\left(* 10^{3} / \mu \mathrm{L}\right)$ & $224[176-269]$ & $190[157-251]$ & $0.084^{*}$ \\
\hline Pre-NLR & $2.89[1.78-4.61]$ & $2.27[1.50-2.85]$ & $0.037^{\star \star}$ \\
\hline Post-NLR & $4.75[3.27-7.04]$ & $6.08[5.46-9.25]$ & $0.001^{\star \star}$ \\
\hline NLRR & $1.69[1.15-2.44]$ & $3.44[2.33-4.10]$ & $<0.001^{\star *}$ \\
\hline \multicolumn{2}{|l|}{ Puncture site, $\mathrm{n}(\%)^{\wedge}$} & & 0.385 \\
\hline Left precornu & $33(32.7)$ & $12(41.4)$ & \\
\hline Right precornu & $68(67.3)$ & $17(58.6)$ & \\
\hline \multicolumn{2}{|l|}{ Initial pressure of vale system, $n(\%)$} & & 0.571 \\
\hline 1.0 & $24(23.8)$ & $6(20.7)$ & \\
\hline 1.5 & $53(52.5)$ & $14(48.3)$ & \\
\hline 2.0 & 19(18.8) & $9(31.0)$ & \\
\hline 2.5 & $5(5.0)$ & $0(0.0)$ & \\
\hline Brain edema around catheter, $n(\%) \Delta$ & $21(20.8)$ & $5(17.2)$ & 0.674 \\
\hline Manipulation of valve system, $n(\%)^{\Lambda}$ & $44(43.6)$ & $10(34.5)$ & 0.382 \\
\hline \multicolumn{4}{|c|}{$\begin{array}{l}\text { Abbreviations: GCS, Glasgow Coma Scale; ICH, spontaneous intracerebral hemorrhage; } \mathrm{LPH} \text {, low } \\
\text { pressure hydrocephalus; NPH, normal pressure hydrocephalus; } \mathrm{HPH} \text {, high pressure hydrocephalus; } \\
\text { Pre-PT, preoperative prothrombin time; Pre-APTT, preoperative activated partial thromboplastin time; } \\
\text { Pre-INR, preoperative international normalized ration; Pre-PLT, preoperative serum thrombocyte; Pre- } \\
\text { NLR, preoperative neutrophil-to-lymphocyte ratio; Post-NLR, postoperative neutrophil-to-lymphocyte } \\
\text { ratio; NLRR, a ratio of post-NLR to pre-NLR. }\end{array}$} \\
\hline \multicolumn{4}{|c|}{$\begin{array}{l}\text { Mann-Whitney U test; }{ }^{\Delta} \text { Pearsons chi-square test; }{ }^{\nabla} \text { Continuous correction chi-square test; }{ }^{口} \text { Fishers } \\
\text { exact test }\end{array}$} \\
\hline \multicolumn{4}{|l|}{$\star P<0.1, * * P<0.05$} \\
\hline
\end{tabular}

\section{Association between elevated NLRR and DICH}


Hypertension, history of craniotomy, preoperative APTT, preoperative PTL, preoperative NLR, postoperative NLR, NLRR $(P<0.1)$ and age, brain edema around catheter, postoperative manipulation of valve system (proposed by previously published articles), were included in the multivariate logistic regression model. The linear relationships between the continuous independent variables and the logit conversation of dependent variable were confirmed by Box-Tidwell test. The indicators of multicollinearity (tolerance, variance inflation factor) were statistically tested, and the results showed that there was no multicollinearity among above independent variables. After adjustment of potential confounding variables, NLRR was considered as an independent risk factors for DICH (odds ratio [OR], 2.774; 95\% confidence interval [Cl], 1.372-5.609; $\mathrm{P}<0.001)$, as well as history of craniotomy $(\mathrm{OR}, 3.505 ; 95 \% \mathrm{Cl}$, 1.040-11.813; $p=0.043$ ) (Table 2).

Table 2

Association between elevated NLRR and DICH secondary to VP shunt

\begin{tabular}{|c|c|c|c|c|}
\hline \multirow{2}{*}{ 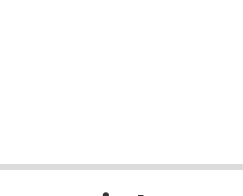 } & \multicolumn{2}{|l|}{ Crude } & \multicolumn{2}{|l|}{ Adjusted } \\
\hline & OR $(95 \% \mathrm{Cl})$ & $\mathbf{P}$ & OR $(95 \% \mathrm{Cl})$ & $\mathbf{P}$ \\
\hline craniotomy & $3.612(1.356-9.620)$ & 0.010 & $3.505(1.040-11.813)$ & 0.043 \\
\hline pre-NLR & $0.916(0.791-1.061)$ & 0.243 & $1.008(0.722-1.409)$ & 0.961 \\
\hline post-NLR & $1.142(1.025-1.274)$ & 0.016 & $1.137(0.931-1.388)$ & 0.207 \\
\hline NLRR & $2.839(1.843-4.374)$ & $<0.001$ & $2.774(1.372-5.609)$ & 0.005 \\
\hline \multicolumn{5}{|c|}{$\begin{array}{l}\text { Adjusted by age, hypertension, preoperative APTT, preoperative PLT, brain edema around catheter, } \\
\text { postoperative manipulation of valve system. }\end{array}$} \\
\hline
\end{tabular}

\section{Receiver operating characteristic curve analysis}

ROC analysis of NLRR regarding DICH was shown in Fig. 2, area under the curve (AUC) was 0.832 , with a $95 \% \mathrm{Cl} 0.754-0.910(\mathrm{P}<0.001)$. The optimal cut off point of NLRR as a predictor for DICH was determined as 2.05 , and the sensitivity was $89.7 \%$, the specificity was $63.4 \%$, the positive predictive value was $40.6 \%$, the negative predictive value was $95.5 \%$.

\section{Propensity score matching (PSM) analysis}

Considering that there were 10 independent variables included in the multivariate logistic regression analysis, the model might be unstable. PSM analysis method ${ }^{12}$ was conducted to confirm the results. After PSM, the propensity score distributions were similar between the two groups (NLRR $\leq 2.05$ group and NLRR > 2.05 group) (Fig. 3A), and the standardized mean differences were much smaller than before (Fig. 3B). The covariates were generally balanced between two groups. Univariable logistic regression 
analysis showed that the incidence of DICH following VP shunt was much higher in NLRR > 2.05 group than NLRR $\leq 2.05$ group ( $p=0.025)$, and the odds ratio was 11.25 (95\% Cl: $1.35-93.50)$. It was similar to the result obtained from the adjusted multivariable logistic regression analysis before PSM, which was 10.01(95\%Cl: 1.64-61.25; $p=0.013)$ (Table 3).

Table 3

Association between NLRR(>2.05 vs $\leq 2.05)$ and DICH secondary to VP shunt

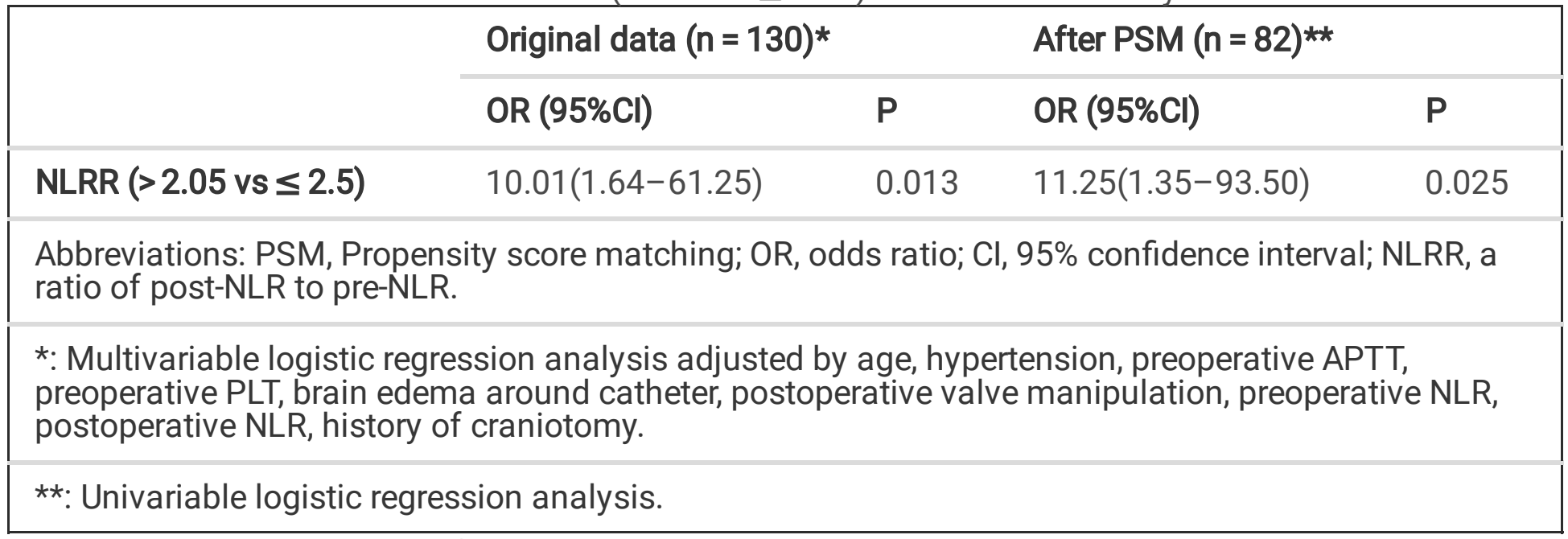

\section{Characteristics of hemorrhage in the patients with DICH}

The mean onset day of DICH after operation was $6.10 \pm 0.53$ day, ranged from 2 day to 13 day. Intraventricular hemorrhage was the most common type, which was presented in 13 patients (44.8\%). 16 patients $(55.2 \%)$ with hematoma volume less than $1 \mathrm{ml}$, only 3 patients $(10.3 \%)$ had hematoma volume more than $15 \mathrm{ml}$, and the maximum hematoma volume was $74.5 \mathrm{ml} .6$ patients (20.7\%) were found to be symptomatic, such as vomiting, epilepsy and decreased consciousness. 15 patients (51.7\%) had a GOS = 3 at the time of discharge, while only one patient (3.4\%) had a GOS $=1$, whose hematoma volume was $74.1 \mathrm{ml}$. The scatter plot of NLRR and hematoma volume was shown in Fig. 4, it seemed that there was no correlation between the NLRR and hematoma volume.

\section{Discussion}

$\mathrm{DICH}$ is one of complications of VP shunt surgery and was first reported in $1985^{13}$. It was considered to be a rare complication with incidence varies from $0.4 \%-4 \% 4,5,14-16$. However, the incidence might be underestimated. Patient with small hematoma might be missed if CT scan was not performed frequently ${ }^{4}$. An article published in 2017 reported that the incidence of postoperative DICH was $7.8 \%$ $(17 / 218)^{17}$. In our study, the incidence was $17.5 \%$, which was much higher than previous studies. Another article published in 2018 reported that the incidence was $23.7 \%$ (34/143) without excluding patients with anticoagulant and antiplatelet therapy ${ }^{18}$. Just as our study, they included the patients with hematoma 
volume less than $1 \mathrm{ml}$ which would be ignored easily and maybe it is the reason. The incidence of symptomatic DICH was $3.6 \%(6 / 166)$ in our study.

The mechanisms underlying the DICH secondary to VP shunt are still controversial. Several hypotheses have been proposed: (1) erosion of cerebral vasculature by the insertion of catheter; (2) fragility of cerebral tissue caused by advanced age, craniotomy, trauma or stroke; (3) disseminated intravascular coagulation (DIC) induced by VP shunt; (4) coagulopathy, anticoagulant or antiplatelet therapy; (5) sudden change of intracranial pressure after manipulation of the valve system ${ }^{7,19}$. Savitz and Bobroff ${ }^{5}$ pointed out that the mechanism of DICH was more likely erosion of surface or deeper small vessel by catheter. This opinion was supported by most reports because most hematomas located along the catheters ${ }^{4}$. The hypercapnia, hypoxia and venous congestion might encourage developing of hematoma at the sites of injury just as the mechanism of traumatic delayed $\mathrm{ICH}^{20}$. A study published in 2017 found that postoperative cerebral edema around the catheter observed on the first CT scan was an independent risk factor for $\mathrm{DICH}^{16}$, which might be a sign of vascular erosion. Nevertheless, this difference was not found in our study $(P=0.674)$. Advanced age, history of craniotomy were considered to be the risk factors for DICH secondary to VP shunt in several articles ${ }^{15-18}$, and these factors might increase the fragility of cerebral tissue. However, one article published in 2017 reported that there was no difference between two groups with respect to age ${ }^{17}$. In our study, we also found that history of craniotomy was an independent risk factor for $\mathrm{DICH}$, while advanced age was not. DIC induced by catheter insertion was considered to be another potential mechanism of DICH. Two cases of DIC associated with VP shunt were reported ${ }^{21,22}$. A Korean study found that prolonged partial thromboplastin time was major risk factor of $\mathrm{DICH}^{18}$. Some studies showed that dual antiplatelet therapy and postoperative anticoagulation therapy would increase the risk of $\mathrm{DICH}^{17,23}$. As for our study, we excluded the patients with anticoagulant or antiplatelet therapy in order to control the confounding factors, and we found that preoperative PT, APTT, INR and PLT were not risk factors for DICH. Two articles presented that postoperative manipulation of valve system might be a risk factor for DICH secondary to VP shunt ${ }^{4,15}$, which was not supported $(P=0.382)$ in our study either.

As the main purpose of the study, we concluded that elevated NLRR could independently predict DICH secondary to VP shunt, which suggested that inflammatory responses might play an important role in the development of DICH. Catheters of VP shunt are made of silicones and may not be immunologically inert ${ }^{24}$. A study pointed out that immune response might be elicited by VP shunt in some patients and could lead to shunt malfunctions ${ }^{25}$. Neutrophils and giant cells were found on the surface of catheters by scanning electron microscopy ${ }^{26}$. In view of the above, we hypothesize that the inflammatory responses may arise from the stimulation of catheter as a foreign body. The acute inflammation phase of foreign body reaction against biomaterials is characterized by migration, adhesion, activation of neutrophils and mast cells, and lasts for hours to few days ${ }^{27}$. Just as the process of brain injury ${ }^{28}$, neutrophils' number increase greatly in the peripheral blood and they can enter central nervous system through the damaged blood brain barrier early ${ }^{11}$. Recruitment and infiltration of neutrophils around catheter could induce 
neurotoxicity by following pathways: production of cytotoxic mediators and proinflammatory cytokines, activation of matrix metalloproteinases and increase of oxidate stress ${ }^{29-31}$. The ensuing further destruction of blood brain barrier, cellular swelling and increased permeability of capillary might trigger

cerebral edema and active bleeding ${ }^{32-36}$. Lymphocytes play an important part in the cellular and humoral immune. It was found that autoreactive $T$ cells could promote vascular reconstruction and healing after cerebral trauma ${ }^{37}$. Therefore, increased neutrophils and decreased lymphocytes might induce DICH secondary to VP shunt. We suggest that the patients with NLRR $>2.05$ should be more carefully observed after VP shunt, and perform CT scans more frequently. However, there was no correlation between the NLRR and hematoma volume. The volume of hematoma might be affected by many factors, such as blood pressure, coagulation function and so on. Since limited understanding of the mechanisms of DICH, our finding would also contribute to identify potential preventive and curative strategies.

NLRR, as a new inflammatory parameter, has smaller variation range than NLR, and can roughly represent the change of inflammatory status due to surgery (including anesthesia) and perioperative treatment. Maybe it could be used as predictors of other diseases requiring surgery, such as postoperative rebleeding of $\mathrm{ICH}$ following minimally invasive surgery.

There are several limitations in our study. The first, it is a retrospective study with small sample size, and a quarter of patients were excluded due to incomplete laboratory or radiological data, which may induce potential selection of bias. The second, there were too many variables included in the multivariate logistic regression analysis, the model might be unstable, even though the result was reconfirmed by PSM analysis. The third, postoperative treatments such as hemostatic therapy were not included in our study, which might be confounding factors. Finally, preoperative neutrophils and lymphocytes were collected within 5 days before surgery. In general, patient's condition was stable before operation, fever or other unusual situation would lead to cancellation of operation, and the laboratory indexes would not change greatly in these days. However, there were still small deviations in these data and they could not represent the preoperative inflammatory status accurately. NLRR's predictive value should be verified by further larger prospective studies.

\section{Conclusions}

In this study, we proposed a new parameter named NLRR, and suggested that DICH following VP shunt was not a rare complication. History of craniotomy and elevated NLRR were independent risk factors for DICH secondary to VP shunt. According to the results, we proposed that inflammatory responses might play an important role in the developing of $\mathrm{DICH}$. More attention should be paid to the patients with NLRR $>2.05$ after VP shunt.

\section{Abbreviations}

DICH = delayed intracerebral hemorrhage; VP = ventriculoperitoneal; NLRR = a ratio of postoperative neutrophil-to-lymphocyte ratio to preoperative neutrophil-to-lymphocyte ratio; PSM = propensity score 
matching; $\mathbf{O R}=$ odds ratio; $\mathbf{C l}=$ confidence interval; $\mathbf{R O C}=$ Receiver operating characteristic; $\mathbf{A U C}=$ area under the curve; NLR = neutrophil-to-lymphocyte ratio; $\mathbf{G C S}$ = Glasgow Coma Scale; GOS = Glasgow outcome scale; $\mathbf{I C H}$ = spontaneous intracerebral hemorrhage; IQR = interquartile range; INR = International normalized ration; $\mathbf{P T}=$ prothrombin time; $\mathbf{A P T T}=$ activated partial thromboplastin time; $\mathbf{P L T}=$ thrombocytes

\section{Declarations}

\section{Ethics approval and consent to participate:}

Participant data were retrospectively reviewed and deidentified. Because of anonymization, consent was waived. The study was approved by our institutional ethics committee (Approval Number: KY2020PJ111).

\section{Consent for publication:}

Not applicable.

\section{Availability of data and materials:}

All data are available within the text of the article. Further anonymized data could be made available to qualified investigators upon reasonable request.

\section{Competing interests:}

The authors declare that they have no competing interests.

\section{Funding:}

None.

\section{Authors' contributions:}

SL designed and conceptualized study, data analysis, drafted manuscript and figures. PC collected the data. HW and FL reviewed CT scans. MC critically revised the manuscript. All authors read and approved the final manuscript.

\section{Acknowledgements:}

We would like to acknowledge the patients and their families.

\section{References}

1. Mallucci CL, Jenkinson MD, Conroy EJ, et al. Antibiotic or silver versus standard ventriculoperitoneal shunts (BASICS): a multicentre, single-blinded, randomised trial and economic evaluation. The Lancet. 2019;394:1530-9. 
2. Musali SR, Manne S, Beniwal HK, Butkuri N, Gollapudi PR, Nandigama PK. Delayed Intracerebral Hemorrhage after Placement of a Ventriculoperitoneal Shunt in a Case of Hydrocephalus: A Rare Case Report and Review of Literature. J Neurosci Rural Pract. 2019;10:533-6.

3. Wu Y, Green N, Wrensch M, Zhao S, Gupta N. Ventriculoperitoneal shunt complications in California: 1990 to 2000. Neurosurgery. 2007;61:557-62. discussion $562-553$.

4. Ma L, Chen YL, Yang SX, Wang YR. Delayed Intracerebral Hemorrhage Secondary to Ventriculoperitoneal Shunt: A Case Report and Literature Review. Med (Baltim). 2015;94:e2029.

5. Savitz M, Bobroff L. Low incidence of delayed intracerebral hemorrhage secondary to ventriculoperitoneal shunt insertion. Journal of neurosurgery. 1999;91:32-4.

6. Ko J, Cha S, Choi B, Lee J, Yun E, Choi C. Hemorrhage rates associated with two methods of ventriculostomy: external ventricular drainage vs. ventriculoperitoneal shunt procedure. Neurologia medico-chirurgica. 2014;54:545-51.

7. Mavridis IN, Mitropoulos A, Mantas C, Karagianni A, Vlachos K. Delayed Intraventricular Hemorrhage following a Ventriculoperitoneal Shunt Placement: Exploring the Surgical Anatomy of a Rare Complication. Case Rep Med. 2017;2017:3953248.

8. Zhang F, Qian J, Tao C, et al. Neutrophil to lymphocyte ratio predicts island sign in patients with intracranial hemorrhage. Med (Baltim). 2018;97:e13057.

9. Zahorec R. Ratio of neutrophil to lymphocyte counts-rapid and simple parameter of systemic inflammation and stress in critically ill. Bratislavske lekarske listy. 2001;102:5-14.

10. Lattanzi S, Brigo F, Trinka E, Cagnetti C, Di Napoli M, Silvestrini M. Neutrophil-to-Lymphocyte Ratio in Acute Cerebral Hemorrhage: a System Review. TransI Stroke Res. 2019;10:137-45.

11. Siwicka-Gieroba D, Malodobry K, Biernawska J, et al. The Neutrophil/Lymphocyte Count Ratio Predicts Mortality in Severe Traumatic Brain Injury Patients. Journal of clinical medicine 2019;8.

12. Lee J, Little TD. A practical guide to propensity score analysis for applied clinical research. Behav Res Ther. 2017;98:76-90.

13. Matsumura A, Shinohara A, Munekata K, Maki Y. Delayed intracerebral hemorrhage after ventriculoperitoneal shunt. Surgical neurology. 1985;24:503-6.

14. Zhou F, Liu Q, Ying G, Zhu X. Delayed intracerebral hemorrhage secondary to ventriculoperitoneal shunt: two case reports and a literature review. International journal of medical sciences. 2012;9:657.

15. Gong W, Xu L, Yang P, et al. Characteristics of delayed intracerebral hemorrhage after ventriculoperitoneal shunt insertion. Oncotarget. 2017;8:42693-9.

16. Guo L, Chen X, Yu B, Shen L, Zhang X. Delayed Intracerebral Hemorrhage Secondary to Ventriculoperitoneal Shunt: A Retrospective Study. World Neurosurg. 2017;107:160-7.

17. Qian Z, Gao L, Wang K, Pandey S. Delayed Catheter-Related Intracranial Hemorrhage After a Ventriculoperitoneal or Ventriculoatrial Shunt in Hydrocephalus. World neurosurgery. 2017;107:84651. 
18. Jang SY, Kim CH, Cheong JH, Kim JM. Risk Factors of Delayed Intracranial Hemorrhage Following Ventriculoperitoneal Shunt. Korean J Neurotrauma. 2018;14:112-7.

19. Snow R, Zimmerman R, Devinsky O. Delayed intracerebral hemorrhage after ventriculoperitoneal shunting. Neurosurgery. 1986;19:305-7.

20. Fujioka S, Matsukado Y, Kaku M, Yano T, Yoshioka S. [Delayed apoplexy following ventricular puncture-a case report]. No shinkei geka Neurological surgery. 1982;10:955-8.

21. Frazier JL, Bova GS, Jockovic K, Hunt EA, Lee B, Ahn ES. Disseminated intravascular coagulation associated with ventriculoperitoneal shunt surgery. J Neurosurg Pediatr. 2010;5:306-9.

22. Shurin S, Rekate H. Disseminated intravascular coagulation as a complication of ventricular catheter placement. Case report. Journal of neurosurgery. 1981;54:264-7.

23. Hudson JS, Nagahama Y, Nakagawa D, et al. Hemorrhage associated with ventriculoperitoneal shunt placement in aneurysmal subarachnoid hemorrhage patients on a regimen of dual antiplatelet therapy: a retrospective analysis. J Neurosurg. 2018;129:916-21.

24. Goldblum R, Pelley R, O'Donell A, Pyron D, Heggers J. Antibodies to silicone elastomers and reactions to ventriculoperitoneal shunts. Lancet. 1992;340:510-3.

25. VandeVord P, Gupta N, Wilson R, et al. Immune reactions associated with silicone-based ventriculoperitoneal shunt malfunctions in children. Biomaterials. 2004;25:3853-60.

26. Gower D, Lewis J, Kelly D. Sterile shunt malfunction. A scanning electron microscopic perspective. Journal of neurosurgery. 1984;61:1079-84.

27. Klopfleisch R, Jung F. The pathology of the foreign body reaction against biomaterials. Journal of biomedical materials research Part A. 2017;105:927-40.

28. Nejat F, Yazdani S, Mahdavi A, et al. Effect of shunt catheter on the systemic immune response: evaluation of neutrophil count, function, and rate of chemotaxis. Journal of neurosurgery. 2007;106:288-91.

29. Chen S, Yang Q, Chen G, Zhang J. An update on inflammation in the acute phase of intracerebral hemorrhage. Translational stroke research. 2015;6:4-8.

30. Liao Y, Liu P, Guo F, Zhang Z, Zhang Z. Oxidative burst of circulating neutrophils following traumatic brain injury in human. PloS one. 2013;8:e68963.

31. Sharma R, Shultz S, Robinson M, et al. Infections after a traumatic brain injury: The complex interplay between the immune and neurological systems. Brain Behav Immun. 2019;79:63-74.

32. Silva Y, Leira R, Tejada J, Lainez J, Castillo J, Dávalos A. Molecular signatures of vascular injury are associated with early growth of intracerebral hemorrhage. Stroke. 2005;36:86-91.

33. Aronowski J, Zhao X. Molecular pathophysiology of cerebral hemorrhage: secondary brain injury. Stroke. 2011;42:1781-6.

34. Wang J. Preclinical and clinical research on inflammation after intracerebral hemorrhage. Progress in neurobiology. 2010;92:463-77. 
35. Zheng H, Chen C, Zhang J, Hu Z. Mechanism and Therapy of Brain Edema after Intracerebral Hemorrhage. Cerebrovasc Dis. 2016;42:155-69.

36. Wang JY, Zhang XT, Wang JQ, et al. Admission Neutrophil-Lymphocyte Ratio Predicts Rebleeding Following Aneurismal Subarachnoid Hemorrhage. World Neurosurg. 2020;138:e317-22.

37. Hofstetter $\mathrm{H}$, Sewell $\mathrm{D}$, Liu F, et al. Autoreactive T cells promote post-traumatic healing in the central nervous system. J Neuroimmunol. 2003;134:25-34.

\section{Figures}




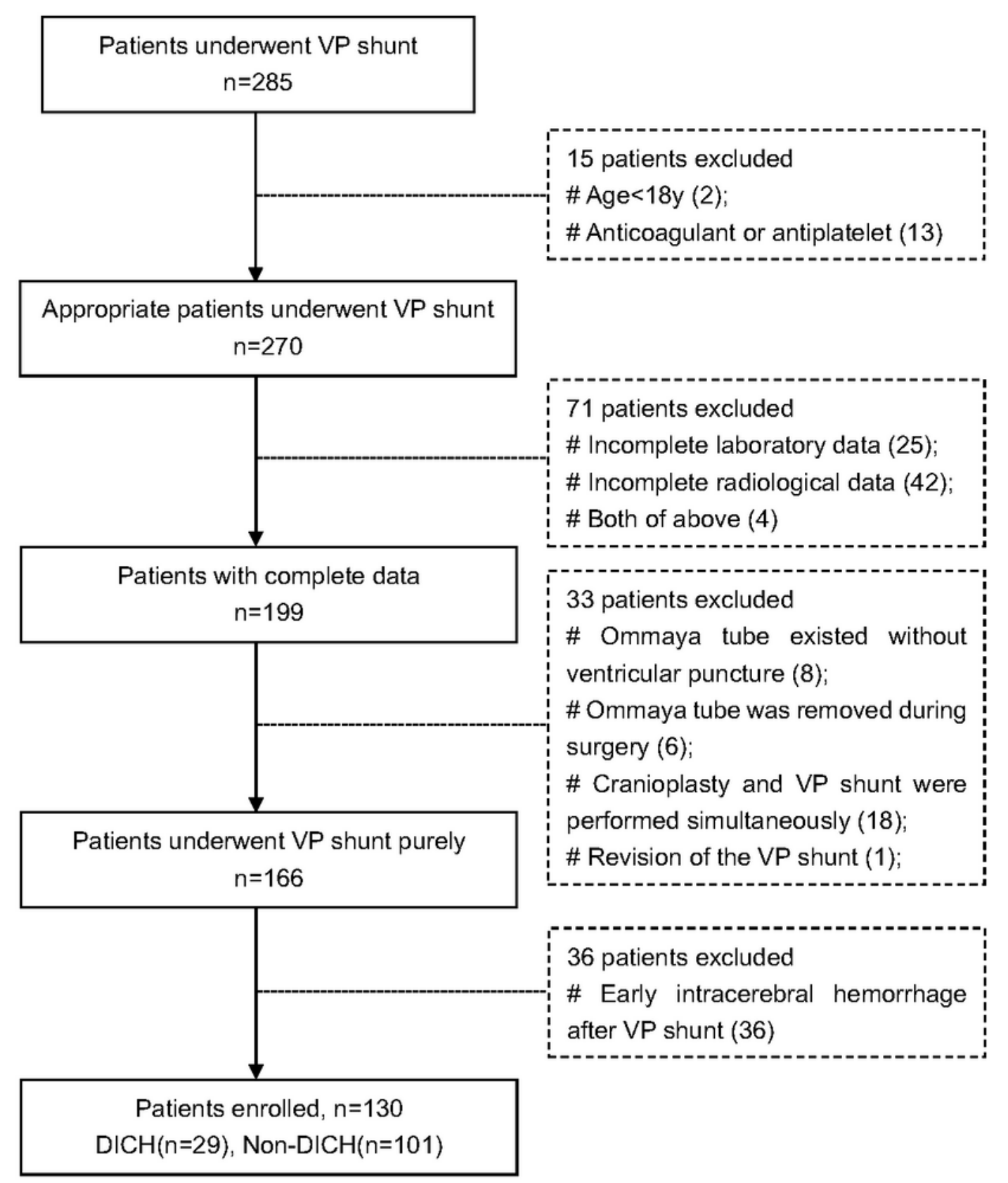

\section{Figure 1}

Flowchart of patient selection 


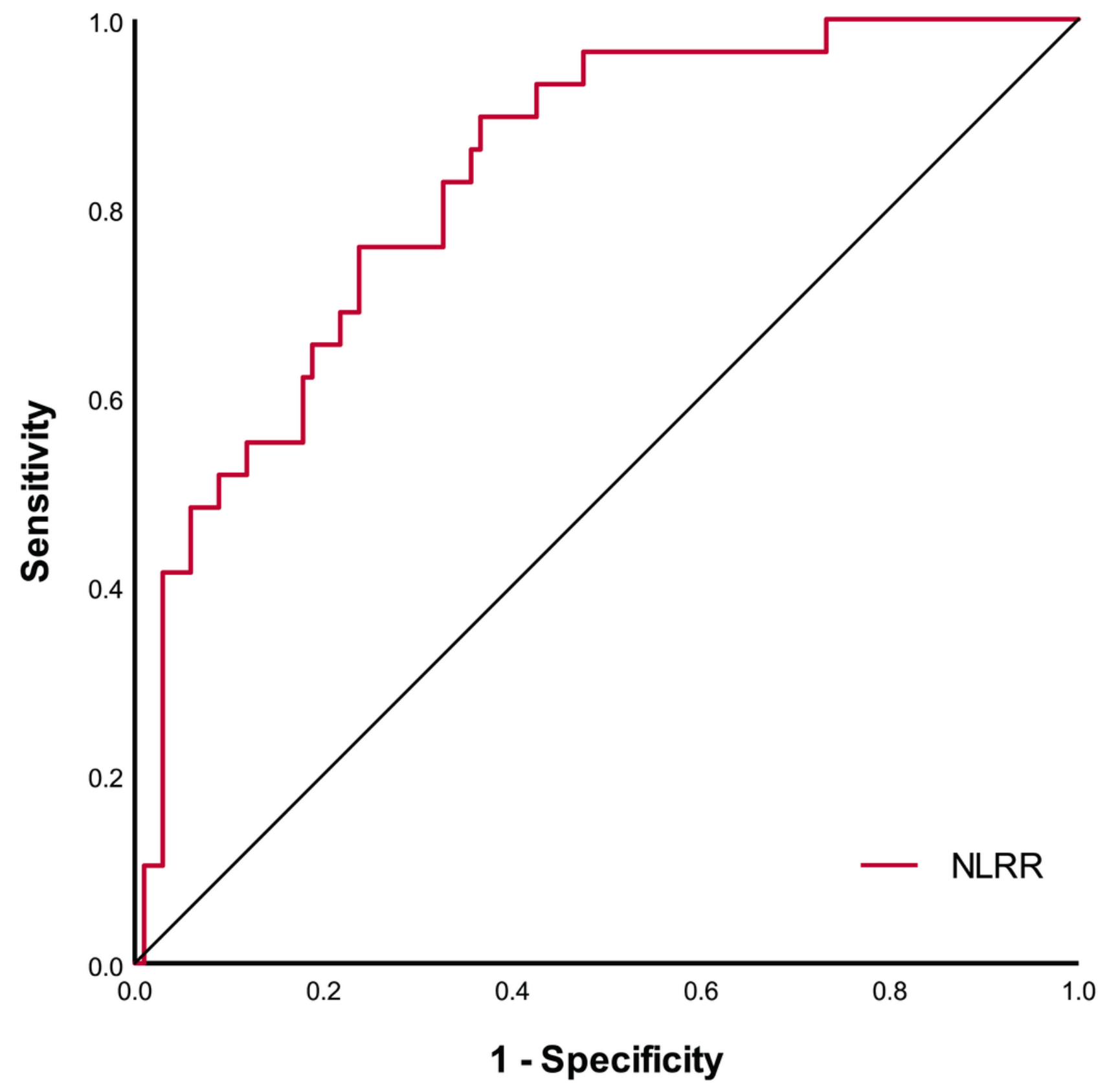

Figure 2

Receiver operating characteristic curves of NLRR to predict DICH Area under the curve was $0.832(95 \% \mathrm{Cl}$ 0.754-0.910; $P<0.001$ ) for NLRR. 
A
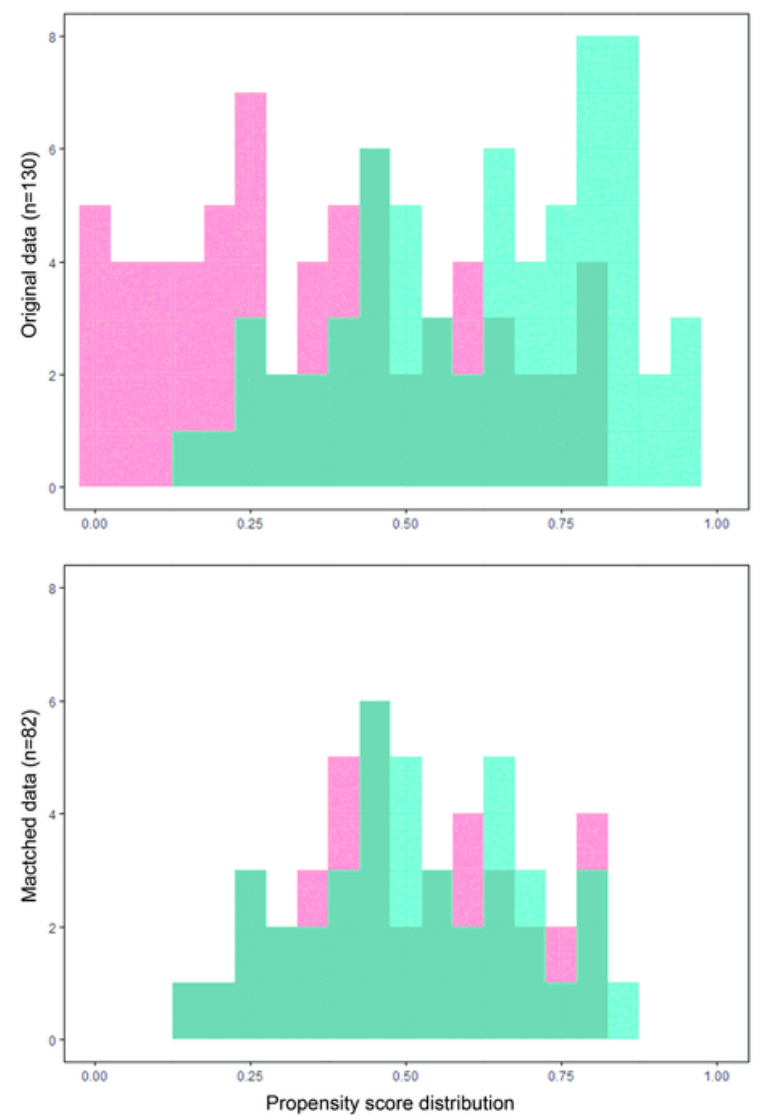

B

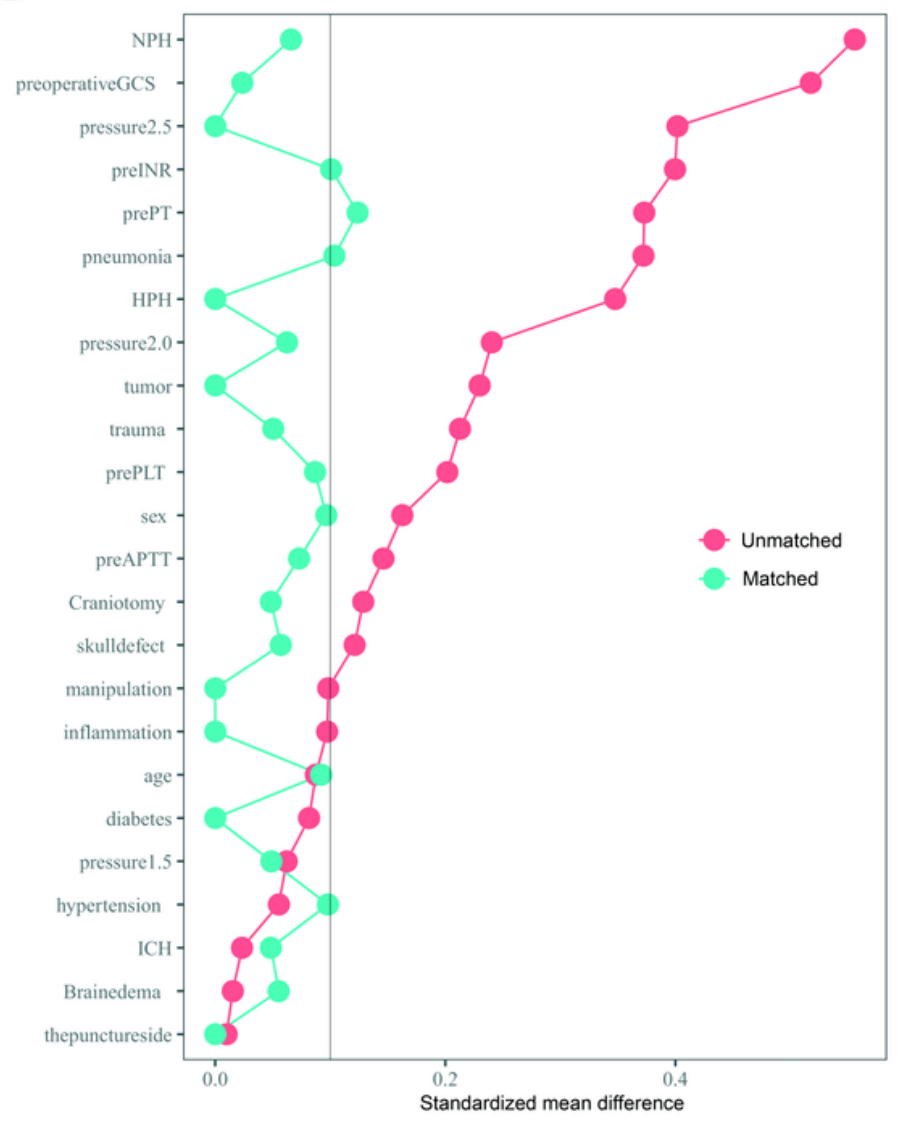

Figure 3

Propensity score distributions and standardized mean differences after PSM Abbreviations: GCS, Glasgow Coma Scale; ICH, spontaneous intracerebral hemorrhage; NPH, normal pressure hydrocephalus; $\mathrm{HPH}$, high pressure hydrocephalus; Pre-PT, preoperative prothrombin time; Pre-APTT, preoperative activated partial thromboplastin time; Pre-INR, preoperative international normalized ration; Pre-PLT, preoperative serum thrombocyte. 

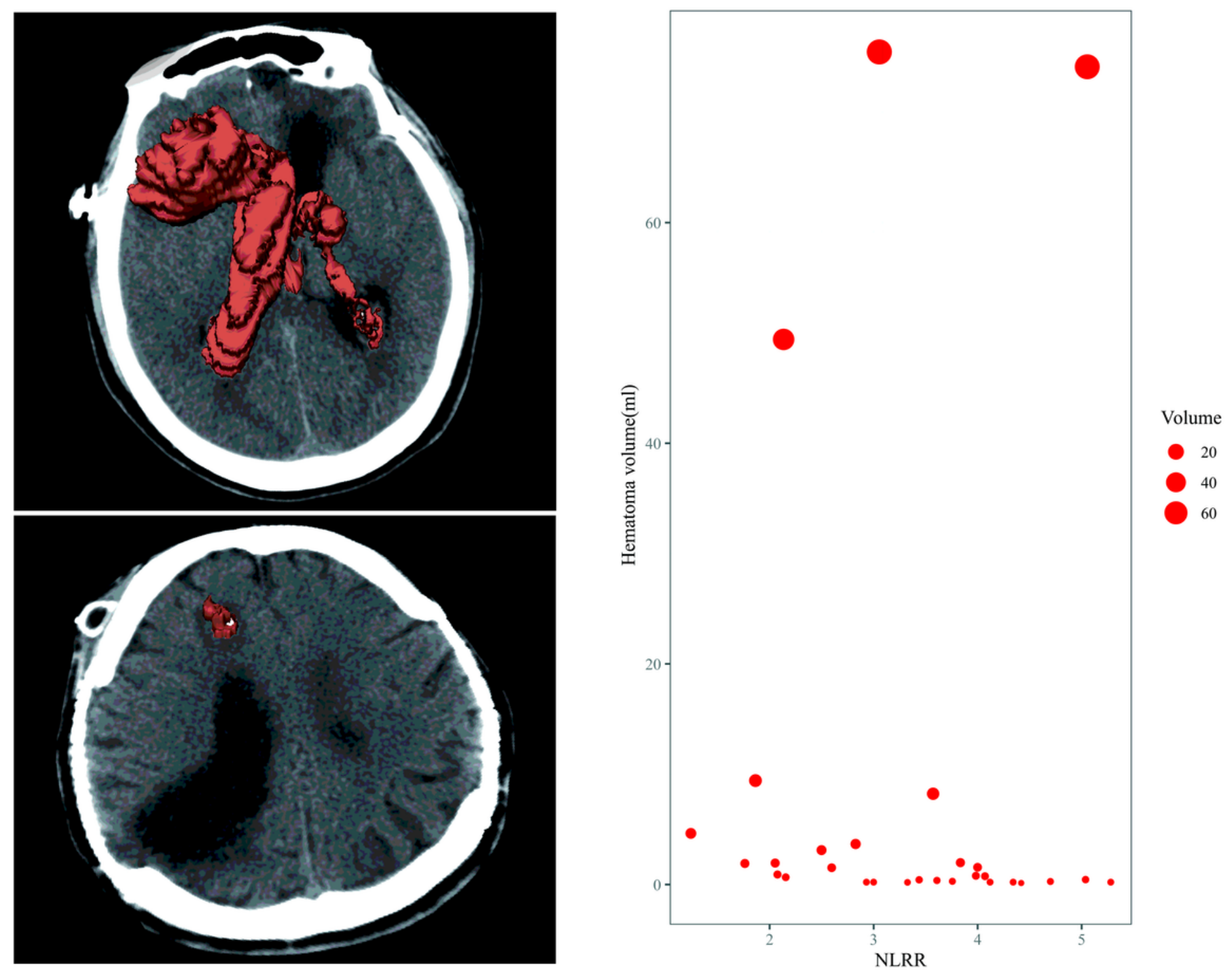

Figure 4

The scatter plot of NLRR value and hematoma volume 